\title{
ETUDE, DES CONCENTRATIONS SANGUINE ET URINAIRE EN CYANURE AU COURS DE PERFUSION DE NITROPRUSSIATE DE SODIUM DE LONGUE DUREE
}

\author{
J. du Cailar,* J.Ci. Mathieu-Daude, J. Kienlen* et P. Chardon*
}

Au coURS DE PRÉCÉdENTES RECHERCHES ${ }^{3.4}$ les concentrations sanguines en thiocyanate $\left(\mathrm{SCN}^{-}\right)$ et cyanure $\left(\mathrm{CN}^{-}\right)$résultant de la dégradation du nitroprussiate de sodium, au cours d'unc hypotension contrôlée en anesthésie, ont été précisées. Le présent travail se propose d'étudier les concentrations sanguines de $\mathrm{CN}^{-}$et son élimination urinaire au cours d'une administra. tion de nitroprussiate de sodium de longue durée.

\section{Materiel d'Etude et Methode}

Le travail porte sur cinq malades (trois femmes et deux hommes) dont laage moyen est de $\mathbf{4 3}$ ans \pm 10 et le poids moyen de $61 \mathrm{~kg} \pm \mathbf{1 2}$. Il s'agit de sujets neurologiques (deux traumatismes crâniens et trois encéphalopathies anoxiques) en coma sévère à distance de l'accident injtial. Le nitroprussiate de sodium est utilisé comme test d'autorégulation de la vasomotricité cérébrale associé à une exploration métabolique cérébrale. Ces sujets sont en nutrition entérale par sonde.

La posologie du nitroprussiate de sodium est

"Département d'Anesthésie-Réanimation (Pr. J. du Caillar), Hôpital Saint-Eloi 34059 Montpellier CEDEX (France).

† Département de Biophysique Médicale (Pr. Ch. Benezech - Pr. J. Liory), Faculté de Médecine de Montpellier (France). de $3 \mu \mathrm{g} \cdot \mathrm{kg}^{-1} \cdot \mathrm{min}^{-1}$, administrés à débit constant pendant 72 heures. La pression artérielle est monitorée par voie sanglante. La valeur de la pression moyenne se stabilise entre 70 et $80 \mathrm{~mm}$ $\mathrm{Hg}$. La diurèse est conservée.

Des prélévements sanguins sont effectués deux heures après le début de la perfusion de NPS puis toutes les 12 heures durant la perfusion et 12 heures et 24 heures après l'arrèt de la perfusion. Sur chaque prélévement sont dosés $\mathrm{CN}^{-}$ globulaire et plasmatique suivant la technique précisée antérieurement. ${ }^{3}$ De ces valeurs est déduite, compte-tenu de l'hématocrite, la valeur de $\mathrm{CN}^{-}$dans le sang total. Le volume urinaire des 24 heures est également noté et la concentration en $\mathrm{CN}^{-}$est mesurée, permettant le calcul de l’élimination urinaire de $\mathrm{CN}^{-}$durant chaque jour de la perfusion et 24 heures après.

Les résultats donnés comportent la moyenne et l'écart sur la moyenne (ESM). La signification est déduite d'un test de comparaison portant sur les données apariées $(p<0,05)$.

\section{Resultars}

Seront envisagées successivement les valeurs du $\mathrm{CN}^{-}$plasmatique, globulaire, total et urinaire.

$\mathrm{CN}^{-}$plasmatique : le tableau I donne les valeurs individuelles et l'évolution des valeurs

TABLEAU I

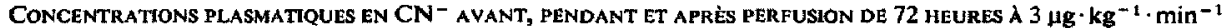
DE NPS CHEZ CINQ PATIENTS. VALEURS MOYENNES ( \pm ESM). T. TEST SUR SÉriEs aPPaRIÉES

\begin{tabular}{|c|c|c|c|c|c|c|c|c|c|c|}
\hline \multirow[b]{2}{*}{$\mathrm{CN}^{-} \mathrm{mg} \cdot 1^{-1}$} & \multirow{2}{*}{$\frac{\begin{array}{c}\text { Avant } \\
\text { NPS }\end{array}}{0}$} & \multicolumn{7}{|c|}{ Pendant NPS } & \multicolumn{2}{|c|}{ Après NPS } \\
\hline & & $2 \mathrm{~h}$ & $12 \mathrm{~h}$ & $24 \mathrm{~h}$ & $36 \mathrm{~h}$ & $48 \mathrm{~h}$ & $60 \mathrm{~h}$ & $72 \mathrm{~h}$ & $12 \mathrm{~h}$ & $24 \mathrm{~h}$ \\
\hline $\begin{array}{l}\text { Bla. } \\
\text { Gom, } \\
\text { Cuc. } \\
\text { Rod. } \\
\text { Jab. } \\
\text { Moy. } \\
\pm \text { ESM }\end{array}$ & $\begin{array}{l}0,010 \\
0,011 \\
0,015 \\
0,005 \\
0,002 \\
0,009 \\
0,002\end{array}$ & $\begin{array}{l}0,026 \\
0,016 \\
0,019 \\
0,020 \\
0,004 \\
0,017 \\
0,004\end{array}$ & $\begin{array}{l}0,020 \\
0,019 \\
0,020 \\
0,022 \\
0,004 \\
0,017 \\
0,003\end{array}$ & $\begin{array}{l}0,016 \\
0,020 \\
0,017 \\
0,010 \\
0,004 \\
0,013 \\
0,003\end{array}$ & $\begin{array}{l}0,014 \\
0,024 \\
0,028 \\
0,007 \\
0,003 \\
0,015 \\
0,005\end{array}$ & $\begin{array}{l}0,020 \\
0,026 \\
0,015 \\
0,007 \\
0,002 \\
0,014 \\
0,004\end{array}$ & $\begin{array}{l}0,019 \\
0,022 \\
0,018 \\
0,009 \\
0,002 \\
0,014 \\
0,004\end{array}$ & $\begin{array}{l}0,024 \\
0,022 \\
0,030 \\
0,010 \\
0,003 \\
0,018 \\
0,005\end{array}$ & $\begin{array}{l}0,013 \\
0,006 \\
0,024 \\
0,008 \\
0,002 \\
0,011 \\
0,0041\end{array}$ & $\begin{array}{l}0,007 \\
0,006 \\
0,020 \\
0,004 \\
0,003 \\
0,008 \\
0,003\end{array}$ \\
\hline Séries appariées & & & $p>0$ & $\overrightarrow{0.6}$ & & & $p>0,0$ & $\begin{array}{l}1,7 \\
(\mathrm{~N} . S .)\end{array}$ & & \\
\hline
\end{tabular}

Canad. Anaesth. Soc. J., vol. 26, no. 5. September 1979 
TABLEAU II

Concentrations Érythrocytaires en CN- AVANT, PENDant et aprés perfusion de 72 heures A $3 \mathrm{\mu g} \cdot \mathrm{kg}^{-1} \cdot \mathrm{min}^{-1}$ DE NPS CHEz CINQ PATIENTS. VALEURS MOYENNES ( \pm ESM). T. TEST SUR SÉRIES APPARIÉES

\begin{tabular}{|c|c|c|c|c|c|c|c|c|c|c|}
\hline \multirow[b]{2}{*}{$\mathrm{CN}^{-} \mathrm{mg} \cdot 1^{-1}$} & \multirow{2}{*}{$\frac{\begin{array}{c}\text { Avant } \\
\text { NPS }\end{array}}{0}$} & \multicolumn{7}{|c|}{ Pendant NPS } & \multicolumn{2}{|c|}{ Après NPS } \\
\hline & & $2 \mathrm{~h}$ & $12 \mathrm{~h}$ & $24 \mathrm{~h}$ & $36 \mathrm{~h}$ & $48 \mathrm{~h}$ & $60 h$ & $72 \mathrm{~h}$ & $12 \mathrm{~h}$ & $24 h$ \\
\hline $\begin{array}{l}\text { Bla. } \\
\text { Gom. } \\
\text { Cuc. } \\
\text { Rod. } \\
\text { Jab. } \\
\text { May. } \\
\pm \text { ESM }\end{array}$ & $\begin{array}{l}0,10 \\
0,06 \\
0,16 \\
0,06 \\
0,02 \\
0,080 \\
0,024\end{array}$ & $\begin{array}{l}0,22 \\
0,07 \\
0,23 \\
0,13 \\
0,13 \\
0,156 \\
0,030\end{array}$ & $\begin{array}{l}0,18 \\
0,20 \\
0,46 \\
0,22 \\
0,23 \\
0,258 \\
0,051\end{array}$ & $\begin{array}{l}0,14 \\
0,36 \\
0,64 \\
0,23 \\
0,28 \\
0,330 \\
0,085\end{array}$ & $\begin{array}{l}0,22 \\
0,41 \\
0,70 \\
0,25 \\
0,28 \\
0,372 \\
0,088\end{array}$ & $\begin{array}{l}0,28 \\
0,46 \\
0,52 \\
0,20 \\
0,33 \\
0,358 \\
0,059\end{array}$ & $\begin{array}{l}0,24 \\
0,38 \\
0,56 \\
0,23 \\
0,32 \\
0,346 \\
0,060\end{array}$ & $\begin{array}{l}0,30 \\
0,39 \\
0,65 \\
0,21 \\
0,20 \\
0,350 \\
0,083\end{array}$ & $\begin{array}{l}0,16 \\
0,14 \\
0,46 \\
0,12 \\
0,11 \\
0,198 \\
0,066\end{array}$ & $\begin{array}{l}0,08 \\
0,14 \\
0,26 \\
0,09 \\
0,08 \\
0,130 \\
0,034\end{array}$ \\
\hline $\begin{array}{l}\text { Séries } \\
\text { appariées }\end{array}$ & \multicolumn{5}{|c|}{$\begin{array}{l}\longleftarrow t=2,57 \\
t=2,38\end{array}$} & \multicolumn{2}{|c|}{$\begin{array}{l}p>0,05 \text { N.S. } \\
p>0,05 \text { N.S. }\end{array}$} & & $\rightarrow$ & \\
\hline
\end{tabular}

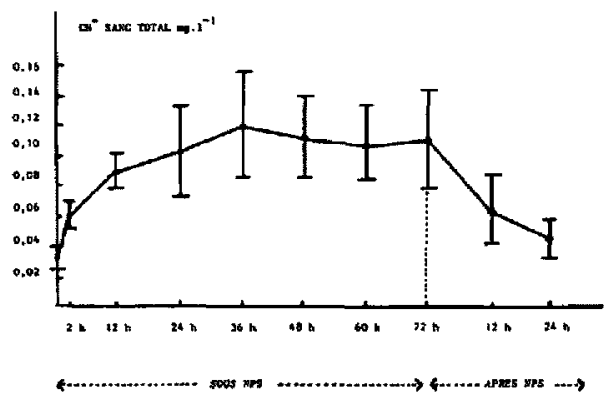

Figure 1 Variations moyennes $\left( \pm \mathrm{ESM}\right.$ ) du $\mathrm{CN}^{-1}$ dans le sang total. Etude chez cing sujets recevant pendant trois jours $3 \mu \mathrm{g} \cdot \mathrm{kg}^{-1} \cdot \mathrm{min}^{-1}$ de NPS. Après NPS a la 12 ème et a la 24 ème heure. la variation notée n'est pas significative par rapport à la valeur avant NPS (test de t sur série appariée).

moyennes. Celles-cj durant les 72 heures de la perfusion et dès la deuxième heure de celle-ci se stabilisent entre 0.012 et $0,022 \mathrm{mg} \cdot \mathrm{l}^{-1}$. On note sur la courbe (Figure 1) deux variations: l'une en baisse de la 12 ème à la 24 ème heures, l'autre en augmentation de la $60 \mathrm{ème}$ à la 72 ème heure. Ces variations ne sont pas significatives. A l'arret de la perfusion. la concentration décroît. A la 12 ème heure, elle présente encore une légère augmentation (non significative) par rapport à la valeur avant perfusion. A la $24 \dot{e} m e$ heure, la valeur initiale est retrouvée.

$\mathrm{CN}^{-}$érythrocytaire : l'étude du tableau II montre que $\mathrm{CN}^{-}$s'accumule dans le globule rouge jusqu à la 36 ème heure où sa concentration moyenne reste sensiblement constante jusqu’à l'arrèt de la perfusion. A l'issue de celle-ci, le taux s'abaisse rapidement. Cependent à la 12 ème

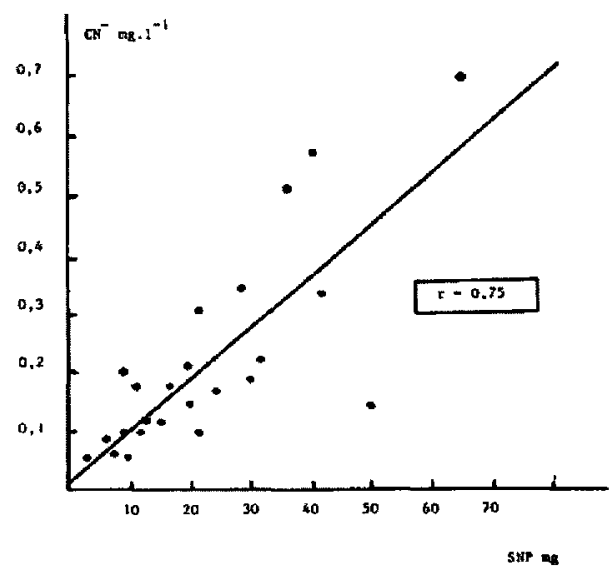

Figure 2 Correlation : concentration sunguine $\mathrm{CN}^{-}$- posologie totale NPS au cours de perfusions de courte durée. Etude chez 24 patients. (D'après J. du Cailar et coll., 1978 (3).

et 24ème heure, on note encore une augmentation (non significative) par rapport à la valeur avant perfusion.

$C N^{-}$sanguin total : le tableau III apporte les valeurs individuelles et la figure 1 objective les fluctuations des moyennes qui refiètent celles du $\mathrm{CN}^{-}$globulaire. En effet à partir de la 36ème heure, la courbe se stabilise autour d'une valeur moyenne de $0,11 \mathrm{mg} \cdot 1^{-1}$. Cependant, à la 12 ème heure, la valeur est déjà de $0.09 \mathrm{mg} \cdot \mathrm{I}^{-1}$ donc très proche de la valeur du plateau. A l'arrêt de la perfusion. les valeurs s'abaissent. Mais à la 24 ème heure, la valeur avant perfusion n'est pas atteinte. 
TABLEAU III

Concentrations dans le sang COMPLet en CN- aVANT, PENDANT et après perfusion de 72 heures À $3 \mu \mathrm{g} \cdot \mathrm{kg}^{-1} \cdot \mathrm{min}^{-1}$ DE NPS CHEZ CINQ PATIENTS. VAleurs MOYeNnes ( \pm ESM). T. TEST SUR SẺries APPARIÉES

\begin{tabular}{|c|c|c|c|c|c|c|c|c|c|c|}
\hline \multirow[b]{2}{*}{$\mathrm{CN}^{-} \mathrm{mg} \cdot \mathrm{1}^{-1}$} & \multirow{2}{*}{$\begin{array}{c}\begin{array}{c}\text { Avant } \\
\text { NPS }\end{array} \\
0\end{array}$} & \multicolumn{7}{|c|}{ Pendant NPS } & \multicolumn{2}{|c|}{ Après NPS } \\
\hline & & $2 \mathrm{~h}$ & $12 \mathrm{~h}$ & $24 \mathrm{~h}$ & $36 \mathrm{~h}$ & $48 \mathrm{~h}$ & $60 \mathrm{~h}$ & $72 \mathrm{~h}$ & $12 \mathrm{~h}$ & $24 \mathrm{~h}$ \\
\hline $\begin{array}{l}\text { Bla. } \\
\text { Gom. } \\
\text { Cuc. } \\
\text { Rod. } \\
\text { Jab. } \\
\text { Moy. } \\
\pm \text { ESM }\end{array}$ & $\begin{array}{l}0,037 \\
0,031 \\
0,056 \\
0,024 \\
0,008 \\
0,031 \\
0,009\end{array}$ & $\begin{array}{l}0,084 \\
0,037 \\
0,078 \\
0,054 \\
0,048 \\
0,060 \\
0,010\end{array}$ & $\begin{array}{l}0,068 \\
0,086 \\
0,139 \\
0,082 \\
0,079 \\
0,091 \\
0,013\end{array}$ & $\begin{array}{l}0,052 \\
0,142 \\
0,198 \\
0,063 \\
0,092 \\
0,109 \\
0,030\end{array}$ & $\begin{array}{l}0,070 \\
0,163 \\
0,223 \\
0,063 \\
0,081 \\
0,120 \\
0,035\end{array}$ & $\begin{array}{l}0,085 \\
0,175 \\
0,161 \\
0,051 \\
0,087 \\
0,112 \\
0,027\end{array}$ & $\begin{array}{l}0,074 \\
0,146 \\
0,175 \\
0,058 \\
0,088 \\
0,108 \\
0,025\end{array}$ & $\begin{array}{l}0,093 \\
0,147 \\
0,204 \\
0,054 \\
0,058 \\
0,111 \\
0,032\end{array}$ & $\begin{array}{l}0,051 \\
0,052 \\
0,146 \\
0,033 \\
0,034 \\
0,063 \\
0,023\end{array}$ & $\begin{array}{l}0,026 \\
0,053 \\
0,087 \\
0,023 \\
0,025 \\
0,043 \\
0,013\end{array}$ \\
\hline $\begin{array}{l}\text { Séries } \\
\text { appariées }\end{array}$ & + & & 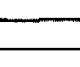 & $\begin{array}{r}\mathrm{t} \\
\mathrm{w}\end{array}$ & 16 & $\begin{array}{l}p> \\
p>\end{array}$ & $5 \mathrm{NS}$ & & $\rightarrow$ & \\
\hline
\end{tabular}

$C N^{-}$inimaire (tableau IV) : l'élimination qui se situe autour de $0,03 \mathrm{mg} \cdot 24 \mathrm{~h}^{-1}$ le premier et le deuxième jour, atugmente à $0.05 \mathrm{mg}$ le troisième jour. Elle se poursuit durant le jour qui suit l'arrét de la perfusion $(0,04 \mathrm{mg})$.

\section{Discussion}

Ce travail confirme l'élévation du $\mathrm{CN}^{-}$sanguin plasmatique et globulaire au cours de la perfusion de NPS. Mais alor's que les précédents travaux et en particulier ceux de Aitken et coll.' confirmés par nos propres recherches (Figure 2), montratient pour des perfusions de courte durée une augmentation de la concentration en $\mathrm{CN}^{-}$proportionnelle à la posologie de NPS, ce travail apporte la notion essentielle qu"à la posologie de $3 \mu \mathrm{g} \cdot \mathrm{kg}^{-1} \cdot \mathrm{min}^{-1}$, correspondant à un apport journalier moyen de $260 \mathrm{mg}$. il s'établit à partir de la 2 ème heure pour le taux de $\mathrm{CN}^{-}$plasmatique et à partir de la 36 ème heure pour le $\mathrm{CN}^{-}$globulaire et le $\mathrm{CN}^{-}$sanguin total un plateau de concentration. Cette constatation confirme le travail de Michenfelder et Tinker ${ }^{-6}$ qui. chez le chien. ont noté au cours d'une perfusion de $0,5 \mathrm{mg} \cdot \mathrm{kg}^{-1} \cdot \mathrm{h}^{-1}$ de NPS lapparition d'un plateatu dans ia concentration en $\mathrm{CN}^{-}$sanguin total à partir de la 30 ème heure. Deux notions peuvent expliquer la stabilisation du $\mathrm{CN}^{-}$sanguin constatée.

(a) une élimination du métabolite peut intervenir. En effet, il existe ainsi que démontré ici mème, une élimination urinaire. Mais celle-ci est au maximum de $0.050 \mathrm{mg} \cdot 24 \mathrm{~h}^{-3}$, valeur négligeable par rapport à la quantité de $\mathrm{CN}^{-}$apportée avec le NPS qui est de $130 \mathrm{mg} \cdot 24 \mathrm{~h}^{-1}$, valeur également réduite par rapport a la disparition en 12 heures, à l'arrét de la perfusion, de $0,24 \mathrm{mg}$ de
$\mathrm{CN}^{-}$contenus par le sang total.* L'élimination urinaire ne peut donc expliquer ni la survenue du plateau de concentration ni l'élimination $d u \mathrm{CN}^{-}$ contenu dans le sang.

(b) une destruction on whe transformation de $C N^{-}$peut également expliquer l'apparition du plateau. Rappelons pour mémoire la classique transformation de $\mathrm{CN}^{-}$en $\mathrm{SCN}^{-}$sous l'iction de la thiosulfate sulfure transférase ainsi que des différentes voies accessoires orientant $\mathrm{CN}^{-}$vers différents composés réactifs ou éliminés par le poumon ou les urines. Dans cette hypothèse, it faut admettre que dans les conditions posologiques de ce travail, à partir de la 30ème heure. l'épuration est égale à l'apport. La part à attribuer, dans cette épuration, aux différents mécanismes possibles reste à préciser. Il est cependant probable que la transformation de $\mathrm{CN}^{-}$en $\mathrm{SCN}^{-}$est le fait essentiel. En effet, un récent travail de Michenfelder et Tinker ${ }^{6}$ plaide en ce sens. Ces auteurs ont en effet démontré, chez le chien. qu'un apport concumitant de thiosulfate (générateurs des radicaux $\mathrm{SH}^{-}$indispensables à l'activité de l'enzyme Rodanèse de Lang) et de NPS abaisse considérablement le taux de $\mathrm{CN}^{-}$et augmente celui de $\mathrm{SCN}^{-}$. Notre étude confirmerait donc que cette enzyme est capable de soutenir régulièrement pendant plusieurs jours son efficacité à la posologie utilisée.

Un deuxième point mérite d'être discuté : il concerne la difference existant entre les valeurs en $\mathrm{CN}^{-}$constatées lors de l'administration du

* Valeur approximative obtenue par calcul à partir de la différence des concentrations sanguines en $\mathrm{CN}^{-}$ notées à la fin de la perfusion et 12 heures après, ramenée à une volémie de cing litres. 


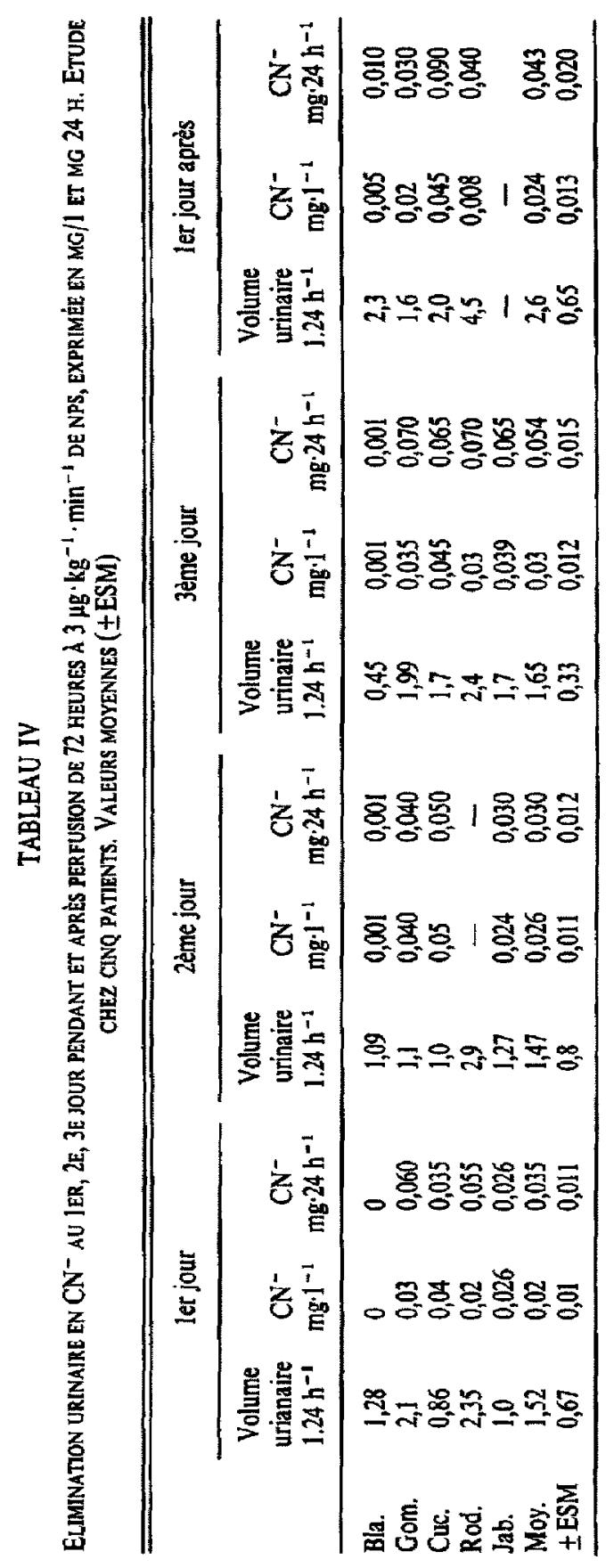


NPS au cours d'une anesthésie et celles notées dans ce travail. En effet au cours de recherches antérieures, ${ }^{3.4}$ nous avons moniré que sous anesthésie générale du type neuroleptanalgésie, à la 2ème heure d'une perfusion de NPS, de valeur moyenne de $3.22 \mu \mathrm{g} \cdot \mathrm{kg}^{-1} \cdot \mathrm{min}^{-1}$. la valeur de $\mathrm{CN}^{-}$sanguin total est de $0,2 \mathrm{mg} \cdot \mathrm{1}^{-1}$ el de 0,3 $\mathrm{mg} \cdot 1^{-1}$ à la 3ème heure. Dans la présente étude pour une posologie, il est vrai, légèrement inférieure - à la 2ème heure on ne note qu'une concentration de $0,06 \mathrm{mg}^{\prime} \mathrm{j}^{-1}$. Cette constatation plaiderait en faveur de la notion que l'anesthésie retarderait l'élimination de $\mathrm{CN}^{-}$.

Différents facteurs peuvent expliquer cette notion : (a) La diurèse est souvent fortement diminuée chez le sujet sous NPS anesthésié, alors qu'elle l'est moins chez le sujet non anesthésiè chez lequel on ne recherche pas une forte hypotension. que nous avons mème évitée dans le cadre de cette étude. Cependant, compte-tenu des considérations précédentes et de la faible élimination urinaire de $\mathrm{CN}^{-}$, la différence de concentrations constatée ne peut pas ètre expliqué par ce facteur. (b) On sait d'autre part que l'anesthésie modifie les circulations régionales et en particulier, qu'elle diminue la circulation splanchnique. D'où un retard éventuel sous anesthésie à la libération de l'enzyme Rhodanèse. Cependant, parmi les différents modes d'anesthésie, il semble que ce soit la neuroleptanalgésie qui perturbe le moins la circulation splanchnique et c'est ce mode d'anesthésie qui avait été employé dans le travail cité plus haut, ${ }^{3,4}$ atténuant donc l'importance possible du facteur circulation. Dans cette optique, rappelons le travail expérimental portant sur le chien de Bethnia et coll. ${ }^{2}$ qui ont démontré que sous anesthésie à I'halothane, le taux de $\mathrm{CN}^{-}$était moins élevé que chez le chien éveillé. Ces résultats, de sens opposé à ceux apportés ici, élargissent le débat. En effet. en dépit des modifications apportées au débit splanchnique par l'halothane, on note avec cet apport une réduction de la concentration en $\mathrm{CN}^{-}$, obligeant Bethnia et coll. ${ }^{2}$ à discuter d'autres hypothèses : fixation sur les protéines sanguines du SNP augmentée retardant sa métabolisation, déviations de diverses activités enzymatiques. $D^{\prime}$ autres recherches seront nécessaires pour conclure cette discussion dont on retiendra cependant la notion que les taux de $\mathrm{CN}^{-}$sanguin peuvent varier selon que le sujet est anesthésié ou non.

On retiendra de ce travail qu'une perfusion de $0,177 \mathrm{mg} \cdot \mathrm{kg}^{-1} \cdot \mathrm{h}^{-1}$ de NPS maintenue pendant trois jours chez l'homme non anesthésié n'en- traîne pas de concentration toxique de $\mathrm{CN}^{-}$dans le sang. En effet, le taux d'équilibre noté est de $0.11 \mathrm{mg}$ alors que les signes de toxicité n'apparaissent que pour des valeurs bien supérieures. ${ }^{1.5}$ Il est cependant possible que des valeurs supérieures de NPS excèderaient les possibilités de détoxification de $\mathrm{CN}^{-}$entrainant son accumulation, une acidose et l'inefficacité de NPS. ${ }^{5.7}$ Cette valeur se situe chez le chien, considéré comme un modèle valable pour l'homme par Michenfelder et Tinker, ${ }^{6}$ au-dessus de 0.5 $\mathrm{mg} \cdot \mathrm{kg}^{-1} \cdot \mathrm{h}^{-1}$. La marge de sécurité en clinique apparait donc large. D'autres travaux sont encore nécessaires chez l'homme pour la définir avec précision en ce qui concerne l'administration de NPS, en longue durée.

\section{RÉSUMÉ}

Chez cinq patients, sujets neurologiques en coma sévère, on a administré à débit constant pendant 72 heures du nitroprussiate de sodium (NPS) à la posologie de $3 \mu \mathrm{g} \cdot \mathrm{kg}^{-1} \cdot \mathrm{min}^{-1}$. Le taux de cyanure $\left(\mathrm{CN}^{-}\right)$sanguin est mesuré deux heures après le début de la perfusion, puis toutes les 12 heures durant la perfusion et 12 heures et 24 heures après la fin de la perfusion. L'élimination urinaire de $\mathrm{CN}^{-}$est aussi étudiée. Les résultats montrent que le taux de $\mathrm{CN}^{-}$sanguin total se stabilise à partir de la $36 \mathrm{e}$ heure à une valeur moyenne de $0,11 \mathrm{mg} / 1$. A l'arrêt de la perfusion, les valeurs s'abaissent, mais à la $24 \mathrm{e}$ heure, la valeur avant perfusion n'est pas atteinte. L'élimination urinaire de $\mathrm{CN}^{-}$, au maximum de $0,050 \mathrm{mg} / 24 \mathrm{~h}$. représente une valeur négligeable qui ne peut expliquer ni l'élimination du $\mathrm{CN}^{-}$ contenu dans le sang, ni la survenue du plateau de concentration. La comparaison des valeurs des concentrations notées au cours des premières heures de perfusion avec celles constatées dans un travail antérieur chez des sujets anesthésiés sous neuroleptanalgésie, montre des valeurs plus basses chez les sujets non anesthésiés. Cette notion est discutée. On retiendra de ce travail qu'une perfusion prolongée dans le temps de SNP à la posologie de $0,177 \mathrm{mg} \cdot \mathrm{kg}^{-1} \cdot \mathrm{h}^{-1} \mathrm{ne} \mathrm{s}^{\prime}$ accompagne pas d'une concentration toxique de $\mathrm{CN}^{-}$ dans le sang.

\section{SUMMary}

Five deeply comatose neurological patients were administered a continuous perfusion of sodium nitroprusside (SNP) at the rate of 3 
$\mu \mathrm{g} \cdot \mathrm{kg}^{-1} \cdot \mathrm{min}^{-1}$. The levels of blood cyanide $\left(\mathrm{CN}^{-}\right)$were measured two hours after the start, then every 12 hours during. and 12 and 24 hours after the end of perfusion. The urinary output of $\mathrm{CN}^{-}$was also studied. The results show that total blood $\mathrm{CN}^{-}$stabilized after 36 hours to a mean value of $0.11 \mathrm{mg} / \mathrm{l}$. When perfusion was stopped, $\mathrm{CN}^{-}$blood levels dropped but did not reach preperfusion values at the 24 th hour. Urinary excretion of $\mathrm{CN}^{-}$which reached a maximum value of $0.050 \mathrm{mg} / 24 \mathrm{~h}$ represents a negligible amount and does not explain the fall of blood $\mathrm{CN}^{-}$and the occurrence of a concentration plateau. The results showing lower values obtained on nonanesthetized patients during the first hours of perfusion compared to those of a previous study done under neuroleptanaesthesia are discussed. These results suggest that prolonged perfusions at SNP at the rate of $0.177 \mathrm{mg} \cdot \mathrm{kg}^{-1} \cdot \mathrm{h}^{-1}$ do not produce toxic blood level of $\mathrm{CN}^{-}$.

\section{BIBLIOGRAPHIE}

1. Aitken. D.. West. D., Smith. T. \& Poznanski. $W$. Cyanide toxicity following nitroprusside in- duced hypotension. Canad. Anaesth. Soc. J. 24 : 65I-660 (1977).

2. BeHNia. R RAMON F CHENG S.C. \& SHARMAD. S. Metabolism of sodium nitroprusside in dogs awake and anesthetised with halothane. Anesthesiology $48: 260-262$ (1978).

3. Callar. J. du. Mathieu-Daude. J.C., DEschodt. J.. Lamarche, Y. \& Castel. J. Nitroprusside. its metabolites and red cell function. Canad. Anaesth. Soc. J. 25 : 92-105 (1978).

4. Calllar. J. du. Mathieu-Daude. J.C. \& DeSCHODT. J. Etude du taux plasmatique et érythrocytaire de l'ion cyanure au cours de la perfusion de nitroprusside de sodium (Nipride). Ann. Anesth. Franç. 17: 169-178 (1977).

5. Grayling, G.W. Miller, E.D. \& Peach, M.J. Sodium cyanide antagonism of vasudilator action of sodium nitroprusside in the isolated rabbit aortic strip. Ancsthesiology $49: 21-25$ (1978).

6. Michenfelder, J.D. \& Tinker. J. Cyanide Ioxicity and thiosulfate protection during chronic administration of sodium nitroprusside in the dog. Anesthesiology $47: 441-448$ (1977).

7. Tremblay, N.A.G.. Davies, D.W. Volgyesi. G., Kadar, D. \& Steward. D.J. Sodium nitroprusside : factors which attenuate its action. Studies with the isolated gracillis muscle of the dog. Canad. Anaesth. Soc. J. $24: 641-650$ (1977). 